Article

\title{
Transcriptome Analysis of the Response of Mature Helicobacter pylori Biofilm to Different Doses of Lactobacillus salivarius LN12 with Amoxicillin and Clarithromycin
}

\author{
Fang Jin (D) and Hong Yang * \\ State Key Laboratory of Microbial Metabolism, School of Life Sciences and Biotechnology, \\ Shanghai Jiao Tong University, Shanghai 201100, China; jinfang@sjtu.edu.cn \\ * Correspondence: hongyang@sjtu.edu.cn; Tel.: +86-021-34205343
}

Citation: Jin, F.; Yang, H. Transcriptome Analysis of the Response of Mature Helicobacter pylori Biofilm to Different Doses of Lactobacillus salivarius LN12 with Amoxicillin and Clarithromycin. Antibiotics 2022, 11, 262. https:// doi.org/10.3390/antibiotics11020262 Academic Editor: Theerthankar Das

Received: 7 January 2022

Accepted: 15 February 2022

Published: 17 February 2022

Publisher's Note: MDPI stays neutral with regard to jurisdictional claims in published maps and institutional affiliations.

Copyright: (C) 2022 by the authors. Licensee MDPI, Basel, Switzerland. This article is an open access article distributed under the terms and conditions of the Creative Commons Attribution (CC BY) license (https:// creativecommons.org/licenses/by/ $4.0 /)$.

\begin{abstract}
Helicobacter pylori is a gastrointestinal pathogen with a high infection rate. Probiotics are clinically used as an adjuvant to improve the cure rate and reduce the side effects of antibiotic treatment for H. pylori. This study is the first to explore the effects of a cell-free supernatant of high- or low-dose Lactobacillus salivarius LN12 combined with amoxicillin (AMX) and clarithromycin (CLR) on H. pylori 3192 biofilms in terms of the biofilm biomass, survival rates, biofilm structure, and transcriptome. The results showed that the combination of the CFS of high-dose LN12 with AMX and CLR had stronger effects on the biofilm biomass, survival rate, and structure of $H$. pylori 3192 biofilms. H. pylori 3192 biofilms may increase the expression of NADH-related genes and downregulate flagellar assembly and quorum sensing-related receptor genes to deal with the stronger stress effects of high-dose LN12 with AMX and CLR. In conclusion, the biofilm biomass, survival rate, structure, and transcriptome results showed that the combination of LN12 CFS with AMX and CLR had dose effects. We recommend that compared with low doses, high doses of L. salivarus LN12 combined with AMX and CLR may be more effective for H. pylori biofilm than low doses.
\end{abstract}

Keywords: Lactobacillus salivarius; Helicobacter pylori; biofilm; transcriptome; dose

\section{Introduction}

As microaerobic Gram-negative bacteria, Helicobacter pylori (H. pylori) can colonize the human stomach through the oral cavity and is closely linked to chronic gastritis, peptic ulcers, gastric mucosa-associated lymphoid tissue lymphoma, and other gastric disorders [1,2]. The global infection rate of $H$. pylori is more than $50 \%$ and affected by geographical and economic conditions, the infection rate in developing countries is higher than that in developed countries, and $H$. pylori was listed as a group I carcinogen by the World Health Organization [3]. The spirochete shape and flagellum structure of H. pylori help it move in the harsh low-pH environment of the stomach, and a variety of adhesion proteins and urease secreted by $H$. pylori facilitate its effective colonization. Two important virulence factors, cytotoxin-associated protein (CagA) and vacuolar cytotoxin (VacA), cause inflammation of gastric epidermal cells [4]. H. pylori can form biofilm structures in vivo and in vitro, which are composed of protein, mannan, LPS-related structures, extracellular DNA, proteins, and outer membrane vesicles. Biofilms can enhance resistance to antibiotics, improve the persistence and survival rate in the host, and reduce the success rate of treatment [5]. At present, the regulatory process of $H$. pylori biofilm formation has not been fully explained, and in particular, transcriptome research on $H$. pylori biofilms remains limited [6-9].

Antibiotics are the first-line therapy for the clinical treatment of $H$. pylori, and triple therapy, consisting of two antibiotics and a proton pump inhibitor, is the most widely 
used [2]. Amoxicillin (AMX) and clarithromycin (CLR) are the most commonly used antibiotics. However, antibiotic resistance is the main challenge in the treatment of H. pylori, as the use of antibiotics leads to the emergence of multidrug-resistant strains, diarrhea, vomiting, other adverse reactions, and changes in the gastrointestinal flora $[2,10]$. Some natural products have been reported to have therapeutic effects on $H$. pylori, such as extracts from Myristica fragrans, Hibiscus rosa sinensis flower, Agrimonia eupatoria, and Fragaria vesca, as have some special nanoparticles (fullerenol nanoparticles, special chitosan/poly acrylic acid particles co-loaded with superparamagnetic iron oxide nanoparticles and amoxicillin, chitosan-based nanoparticles) and N-acetylcysteine (NAC) (when in combination with clarithromycin and lansoprazole) [5,10-17]. Probiotic cell-free supernatant (CFS) can interfere with the growth of H. pylori, destroy H. pylori biofilms, hinder the adhesion of $H$. pylori in the stomach, and inhibit the immune response induced by $H$. pylori $[18,19]$. Compared with natural products, probiotics supplemented with antibiotics can not only improve the cure rate of $H$. pylori but also reduce adverse reactions to antibiotic treatment. However, the type, dose, interval, and treatment time of probiotics can affect the success rate of $H$. pylori treatment [20-22]. At present, there is no unified standard for the dose of clinical probiotics; $10^{8}, 10^{9}$, and $10^{10} \mathrm{CFU}$ are used, and no research has clearly shown that the higher the dose of probiotics in the treatment of $\mathrm{H}$. pylori is, the better the effects will be [21].

At present, no study has explored the effects of the CFS of different doses of probiotics combined with AMX and CLR on H. pylori biofilms, especially on the transcriptome. Our previous studies showed that Lactobacillus salivarius (L. salivarus) LN12 CFS could enhance the destructive effects of AMX and CLR on H. pylori 3192 biofilm in vitro [23]. This study was the first to explore the effects of CFS of different doses of L. salivarus LN12 combined with AMX and CLR on H. pylori 3192 biofilm biomass, survival rate, and structure, and especially on the transcriptome.

\section{Results}

2.1. Effects of CFSs of Different Doses of L. salivarus LN12 in Combination with AMX and CLR on the Biomass of H. pylori Biofilms

After washing twice with PBS, 4-day mature H. pylori 3192 biofilms on coverslips were subjected to different treatments for $12 \mathrm{~h}$, and the biomass was quantified with the crystal violet staining method. The different treatments were as follows: AMX and CLR at FIC concentrations alone (FIC group) or combined with $6.25 \%$ CFS of $10^{5} \mathrm{CFU} / \mathrm{mL}$ (CF5 group), $10^{7} \mathrm{CFU} / \mathrm{mL}$ (CF7 group), or $10^{9} \mathrm{CFU} / \mathrm{mL}$ (CF9 group) L. salivarus LN12. BB2 was used as a negative control. Figure 1 showed that compared to the control group, FIC, CF5, CF7, and CF9 all significantly decreased the biomass $(p \leq 0.05)$, and CFS of different doses of $L$. salivarus LN12 could enhance the destructive effects of FIC on biofilms. With the increasing doses of L. salivarus LN12, the effects of CFS combined with AMX and CLR on biofilms were more significant.

\subsection{Effects of CFSs of Different Doses of L. salivarus LN12 in Combination with AMX and CLR on the Viability of H. pylori Biofilms}

CFSs of different doses of L. salivarus LN12 were combined with AMX and CLR according to the above methods. The survival rate of $H$. pylori 3192 biofilms on coverslips under different treatments was evaluated by counting CFUs on blood plates. Figure 2 showed that the combination of CFSs of different doses of $L$. salivarus LN12 with AMX and CLR decreased the biofilm bacterial survival rate $(p \leq 0.05)$. CFS enhanced the destructive effects of FIC on the biofilm survival rate, and these effects became stronger with increasing doses of L. salivarus LN12. 


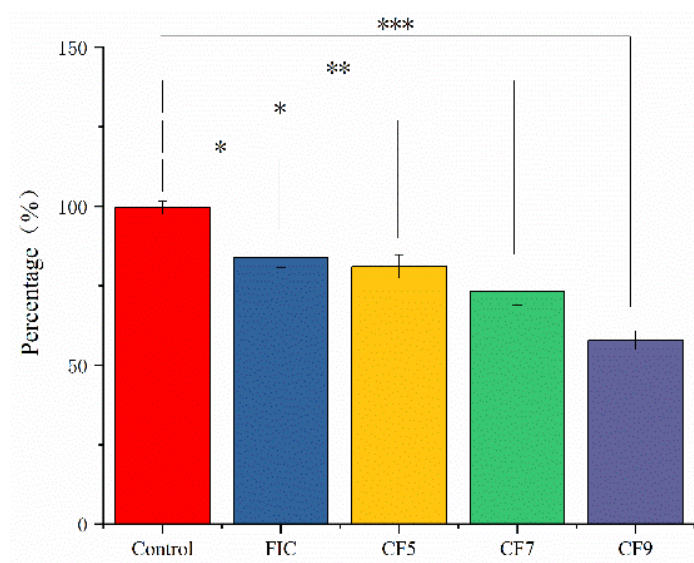

Figure 1. Effects of different treatments on H. pylori 3192 biofilm biomass. Four-day mature H. pylori biofilm was treated with different treatments, and the biomass of the H. pylori biofilm was assessed by $1 \%$ crystal violet staining. Control group: BB2, FIC group: $1 \times$ FIC of AMX and CLR, CF5 group: $6.25 \%$ CFS of $10^{5}$ CFU / mL L. salivarus LN12 in combination with $1 \times$ FIC of AMX and CLR, CF7 group: $6.25 \%$ CFS of $10^{7} \mathrm{CFU} / \mathrm{mL}$ LN12 in combination with $1 \times$ FIC of AMX and CLR, CF9 group: $6.25 \%$ CFS of $10^{9} \mathrm{CFU} / \mathrm{mL}$ L. salivarus LN12 in combination with $1 \times$ FIC of AMX and CLR. AMX: Amoxicillin, CLR: Clarithromycin. $*: p \leq 0.05, * *: p \leq 0.01$, and $* * *: p \leq 0.001$.

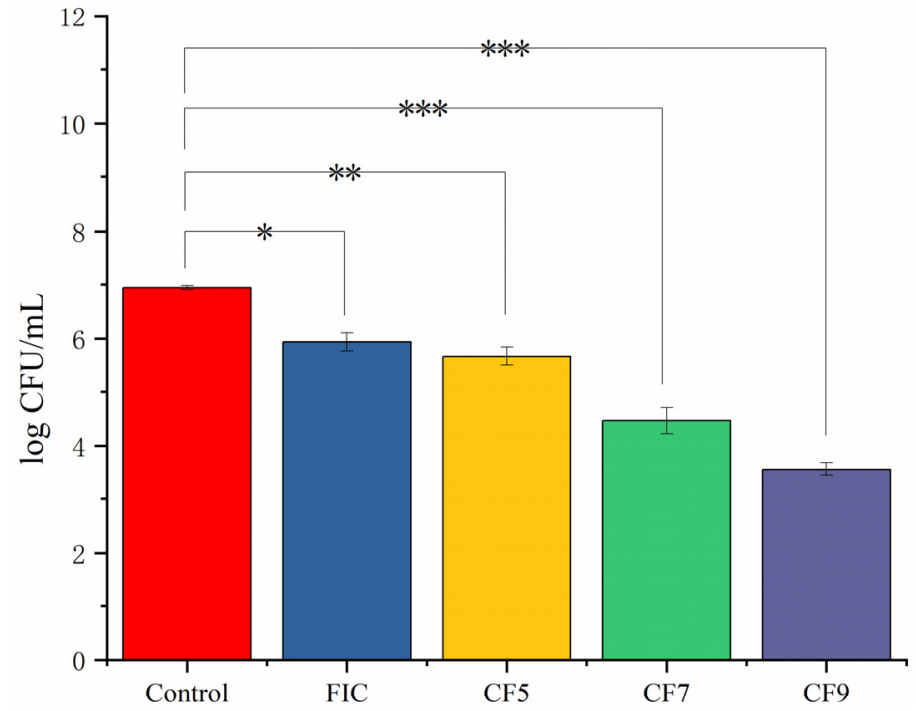

Figure 2. Effects of different treatments on the survival rate of $H$. pylori 3192 biofilm. Four-day mature $H$. pylori biofilm was treated with different treatments, and the survival rate was counted on a blood agar plate after $72 \mathrm{~h}$. Control group: BB2, FIC group: $1 \times$ FIC of AMX and CLR, CF5 group: $6.25 \%$ CFS of $10^{5} \mathrm{CFU} / \mathrm{mL}$ L. salivarus LN12 in combination with $1 \times$ FIC of AMX and CLR, CF7 group: $6.25 \%$ CFS of $10^{7} \mathrm{CFU} / \mathrm{mL}$ L. salivarus LN12 in combination with $1 \times$ FIC of AMX and CLR, CF9 group: $6.25 \%$ CFS of $10^{9} \mathrm{CFU} / \mathrm{mL}$ L. salivarus LN12 in combination with $1 \times$ FIC of AMX and CLR. AMX: Amoxicillin, CLR: Clarithromycin. $*: p \leq 0.05, * *: p \leq 0.01$, and $* * *: p \leq 0.001$.

2.3. Effects of CFSs of Different Doses of L. salivarus LN12 in Combination with AMX and CLR on the Structure of H. pylori Biofilms

The effects of the different treatments on H. pylori 3192 biofilms were observed by scanning electron microscopy (SEM). Figure 3 showed that the biofilm in the control group (A) was dense with a small number of holes, and the bacteria were intertwined and arranged in an orderly way. The bacteria were mainly spiral rod-shaped, accompanied by a small number of spherical shapes, which is consistent with existing literature reports [24]. Compared with the control group (A), the biofilm structure under different treatments (B, C, D, E) was damaged, and the combination of L. salivarus LN12 CFS with AMX and 
CLR enhanced the damage to the biofilm caused by the antibiotics. When combined with AMX and CLR, as the dose of L. salivarus LN12 increased, the proportion of the rod-shaped morphology of bacteria in the biofilm was significantly decreased while the proportion of spherical bacteria increased, more holes appeared, and more fragmentation was observed (especially in the area marked with an arrow in Figure 3).

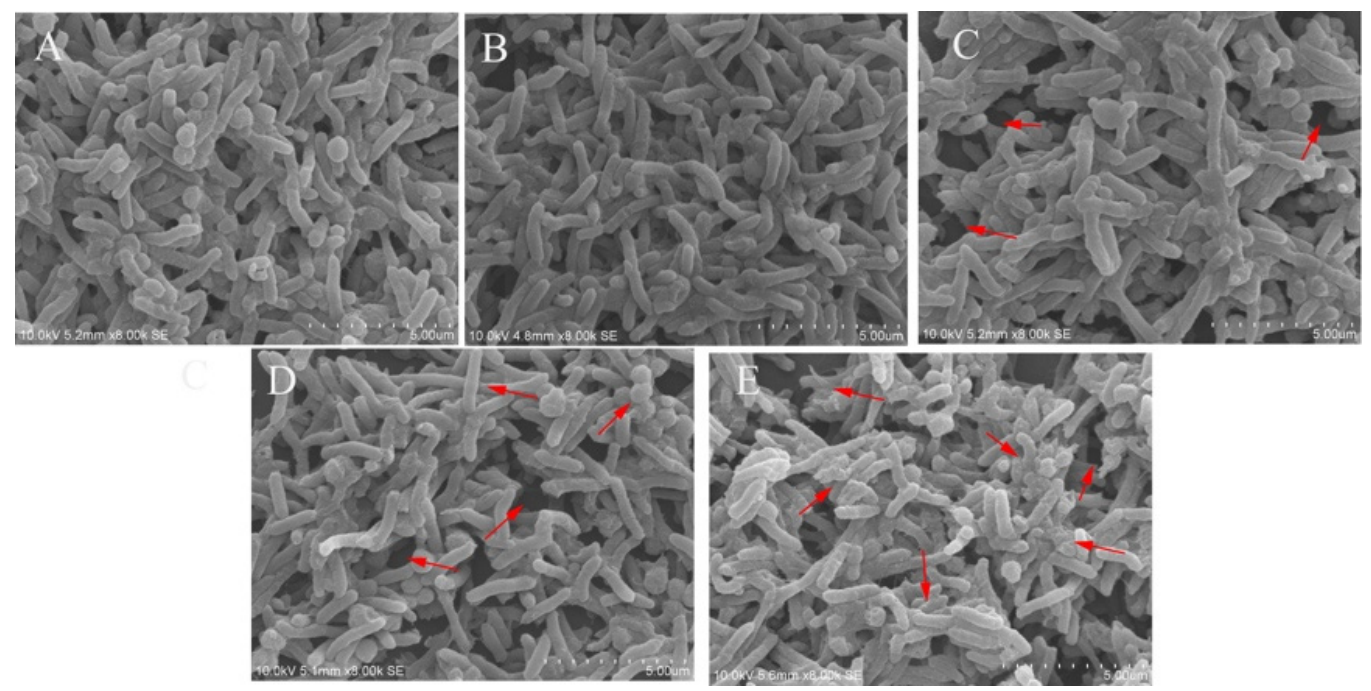

Figure 3. Effects of different treatments on a 4-day mature H. pylori 3192 biofilm under SEM. (A) (Control group): BB2, (B) (FIC group): $1 \times$ FIC of AMX and CLR, (C) (CF5 group): 6.25\% CFS of $10^{5} \mathrm{CFU} / \mathrm{mL}$ L. salivarus LN12 in combination with $1 \times$ FIC of AMX and CLR, (D) (CF7 group): $6.25 \%$ CFS of $10^{7} \mathrm{CFU} / \mathrm{mL}$ L. salivarus LN12 in combination with $1 \times$ FIC of AMX and CLR, (E) (CF9 group): $6.25 \%$ CFS of $10^{9} \mathrm{CFU} / \mathrm{mL}$ L. salivarus LN12 in combination with $1 \times$ FIC of AMX and CLR. Arrows indicated the pores in biofilm and bacterial fragmentation. The magnification is $8000 \times$, the scale bar is $5.00 \mu \mathrm{m}$. AMX: Amoxicillin, CLR: Clarithromycin.

SYTO9 and PI can specifically combine with living and dead bacteria in biofilms. Under confocal laser scanning microscopy (CLSM), living bacteria and dead bacteria are green and red, respectively, under excitation at 488 and $560 \mathrm{~nm}$, enabling visualization of the distribution of bacteria in biofilms (Figure 4). Dense green fluorescent clusters were observed in three views of the control group, and almost no red fluorescence was found in the side view, indicating that the untreated biofilm had a compact structure, and the proportion of dead cells under CLMS was almost zero (Figure 4A). After different treatments, the biofilm structure changed to varying degrees (Figure 4B-E). It can be seen from three angles that the biofilm was dispersed to varying degrees. The combination of L. salivarus LN12 CFS and AMX and CLR can enhance the destructive effects of antibiotics on the biofilm (Figure 4C-E), and the destructive effects increased as the dose of L. salivarus LN12 increased: the biofilm structure became looser, the distribution of green cells was smaller, and the proportion of dead bacteria (red) was larger, which is consistent with the SEM and biofilm viability results. 


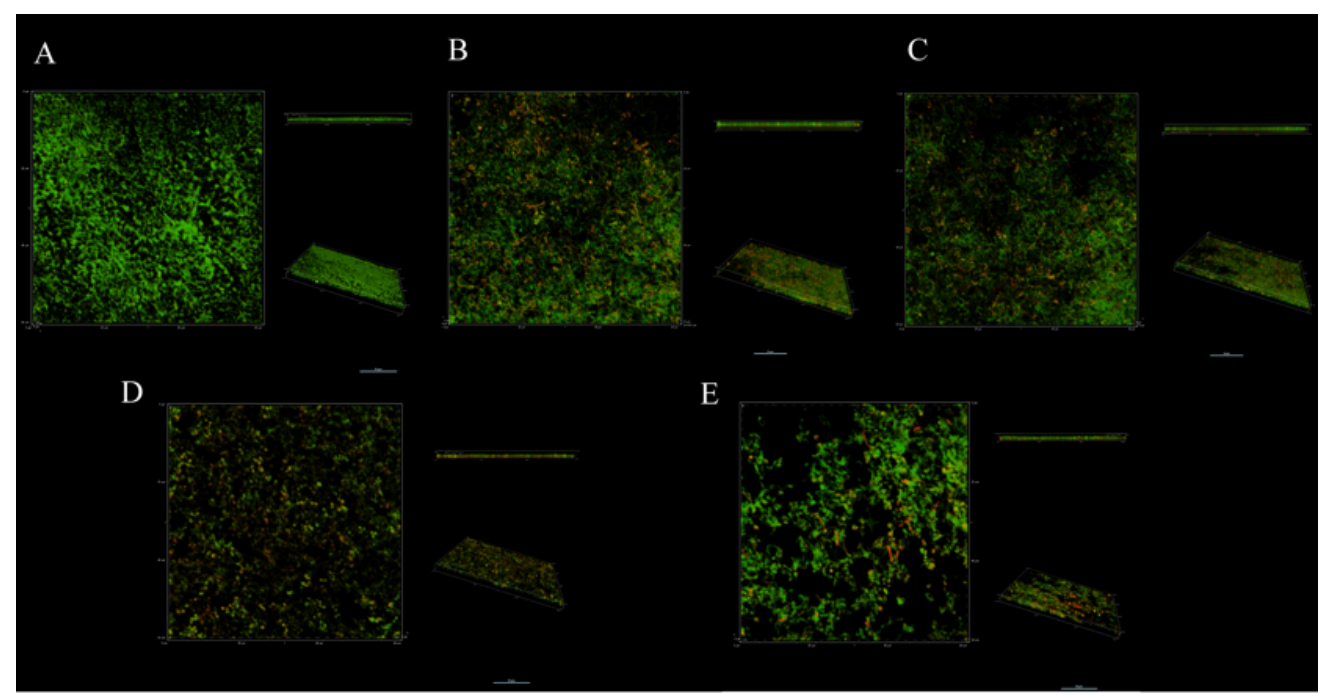

Figure 4. Effects of different treatments on H. pylori biofilm by CLSM. (A) (Control group): BB2, (B) (FIC group): $1 \times$ FIC of AMX and CLR, (C) (CF5 group): $6.25 \%$ CFS of $10^{5} \mathrm{CFU} / \mathrm{mL}$ L. salivarus LN12 in combination with $1 \times$ FIC of AMX and CLR, (D) (CF7 group): $6.25 \%$ CFS of $10^{7} \mathrm{CFU} / \mathrm{mL}$ L. salivarus LN12 in combination with $1 \times$ FIC of AMX and CLR, (E) (CF9 group): $6.25 \%$ CFS of $10^{9} \mathrm{CFU} / \mathrm{mL}$ L. salivarus LN12 in combination with $1 \times$ FIC of AMX and CLR. The scale bar is $10 \mu \mathrm{m}$. Every group contains three views: the main view (observed horizontally along the $x-y$ axis), the side view (observed horizontally along the $\mathrm{x}-\mathrm{z}$ axis), and the view at a certain angle. AMX: Amoxicillin, CLR: Clarithromycin.

\subsection{Transcriptome Analysis}

To explore the effects of CFSs of different doses of L. salivarus LN12 combined with AMX and CLR on the H. pylori 3192 transcriptome, the cell-free supernatants (CFSs) of $10^{7}$ and $10^{9} \mathrm{CFU} / \mathrm{mL}$ L. salivarus LN12 were diluted to concentrations of $6.25 \%$, then combined with FIC of AMX and CLR, and named the low-dose group (CF7) and highdose group (CF9). AMX and CLR at the FIC values were used as the FIC group and BB2 as the blank control group (control). Then, 4-day $H$. pylori 3192 biofilms were treated with FIC, CF7, CF9, and BB2 for $12 \mathrm{~h}$, and the changes in the biofilm transcriptome were observed. The RNA of all samples met the requirements of RNA-Seq (Supplementary Table S1). The density distribution analysis of the transcriptome expression showed that the structure of all groups was basically the same, with most genes showing medium expression (Supplementary Figure S1). The default screening conditions of differentially expressed genes (DEGs) were FDR $\leq 0.05$ and $|\log 2 \mathrm{FC}| \geq 1$, and the correction method for multiple tests was BH (fdr correction with Ben-jamini/Hochberg). The volcano plot (Supplementary Figure S2) showed 694 DEGs, 345 downregulated genes, and 349 upregulated genes in the FIC group compared with the control group; 584 DEGs, 286 downregulated genes, and 298 upregulated genes in the CF7 group compared with the control group; 625 DEGs, 315 downregulated genes, and 310 upregulated genes in the CF9 group compared with the control group; 46 DEGs, 11 downregulated genes, and 35 upregulated genes in the CF7 group compared with the FIC group; 400 DEGs, 190 downregulated genes, and 210 upregulated genes in the CF9 group compared with the FIC group; and 225 DEGs, 121 downregulated genes, and 104 upregulated genes in the CF7 group compared with the $\mathrm{CF} 9$ group. PCA showed that the CF7 group was correlated with the FIC group but presented a different state from the control and CF9 groups (Figure 5). The Venn diagram (B) and heatmap (C) showed that the four groups were in different states but were related to each other. FIC, CF7, and CF9 were different from the control group, but the states of CF7 and FIC were more similar, and the differences between CF9 and FIC were greater. 

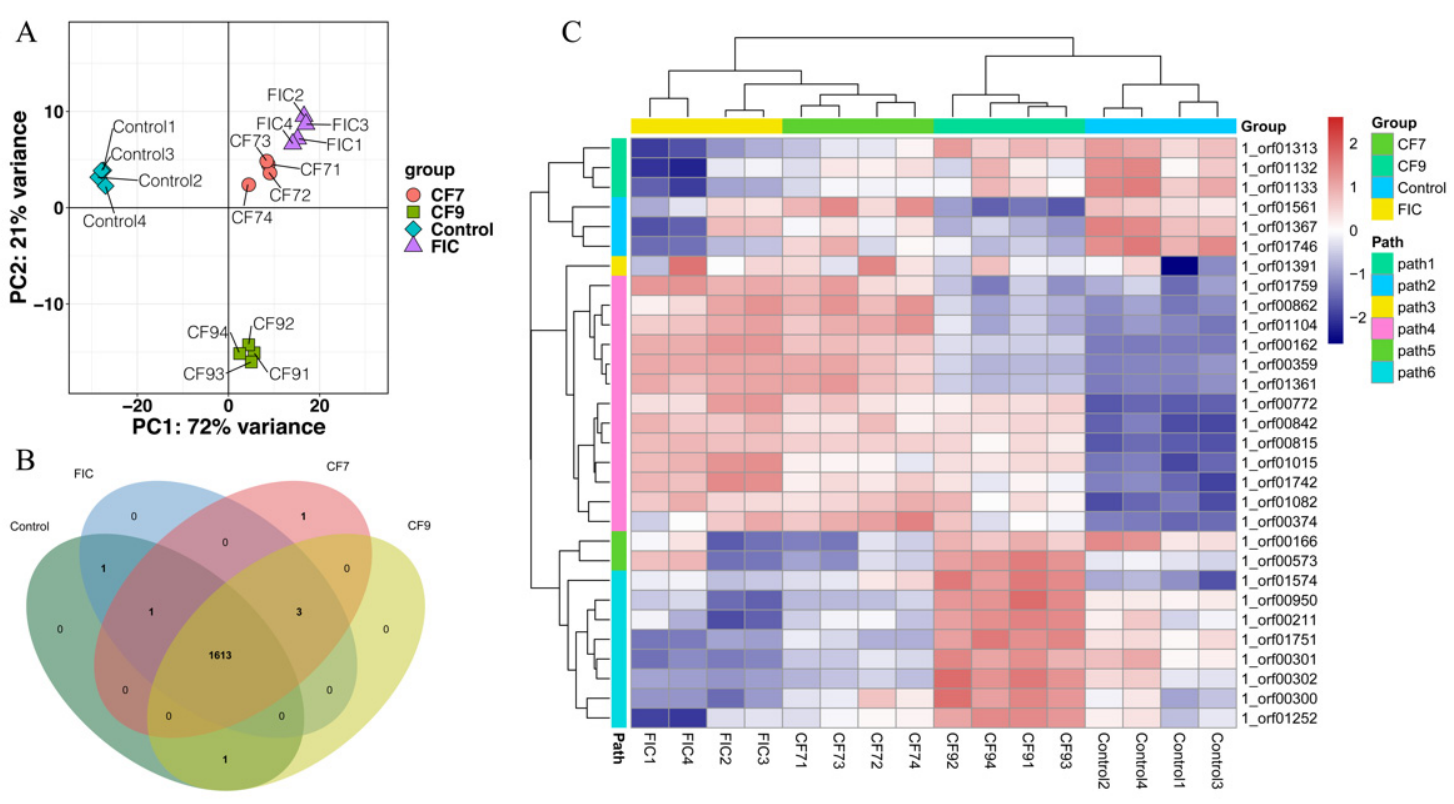

Figure 5. H. pylori 3192 biofilm transcriptome in different treatment groups. Principal component analysis (PCA) (A), Venn diagram (B), and heat map (C) showed the common and differently expressed genes in different treatment groups. Control group: BB2, FIC group: $1 \times$ FIC of AMX and CLR, CF7 group: $6.25 \%$ CFS of $10^{7} \mathrm{CFU} / \mathrm{mL}$ L. salivarus LN12 in combination with $1 \times$ FIC of AMX and CLR, CF9 group: $6.25 \%$ CFS of $10^{9} \mathrm{CFU} / \mathrm{mL}$ L. salivarus LN12 in combination with $1 \times$ FIC of AMX and CLR. Four independent biological samples were used in every group.

\subsubsection{GO Enrichment Analysis}

We focused mainly on the effects of CFSs of different doses of L. salivarus LN12 combined with AMX and CLR on the H. pylori 3192 biofilm transcriptome. In the following section, the AMX- and CLR-treated group (FIC) was used as the control, and CFSs of high or low doses of L. salivarus LN12 combined with AMX and CLR were used as treatment groups (CF7 or CF9). GO functional annotation and enrichment analysis were carried out using the GO database. The top $10 \mathrm{GO}$ terms with the lowest $p$ values in cellular component (CC), molecular function (MF), and biological process (BP) were collected, and the first 5 GO terms were analyzed, as shown in Figure 6. For BP, compared to the FIC group, the most significant GO enrichment terms in the CF7 and CF9 groups were the respiratory electron transport chain and electron transport chain, and the largest numbers of DEGs in CF9 and CF7 were related to the electron transport chain and oxidation-reduction process, respectively. For CC, compared to the FIC group, the most significant GO enrichment terms in the CF7 and CF9 groups were the membrane and integral component of membrane, and CF9 had more DEGs. For MF, compared to the FIC group, the top five GO terms in CF7 and CF9 shared high similarity in oxidoreductase activity acting on NAD(P)H, NADH dehydrogenase activity, and quinone binding. The main difference between CF7 and CF9 was nickel cation binding, which was the third significant GO term in CF9. Furthermore, ClueGo was used to further cluster and visually analyze GO terms with $p<0.1$ in CC, MF, and BP. Figure 7 shows that compared with the FIC group, the CF7 group obtained 11 final KappaScore groups and CF9 obtained 14. The most significant GO terms (color labeled) in each KappaScore group were also different in CF7 and CF9. Overall, compared with the FIC group, CF9 and CF7 shared some GO enrichment terms but also had distinct terms, and CF9 had higher metabolic vigor. 
A

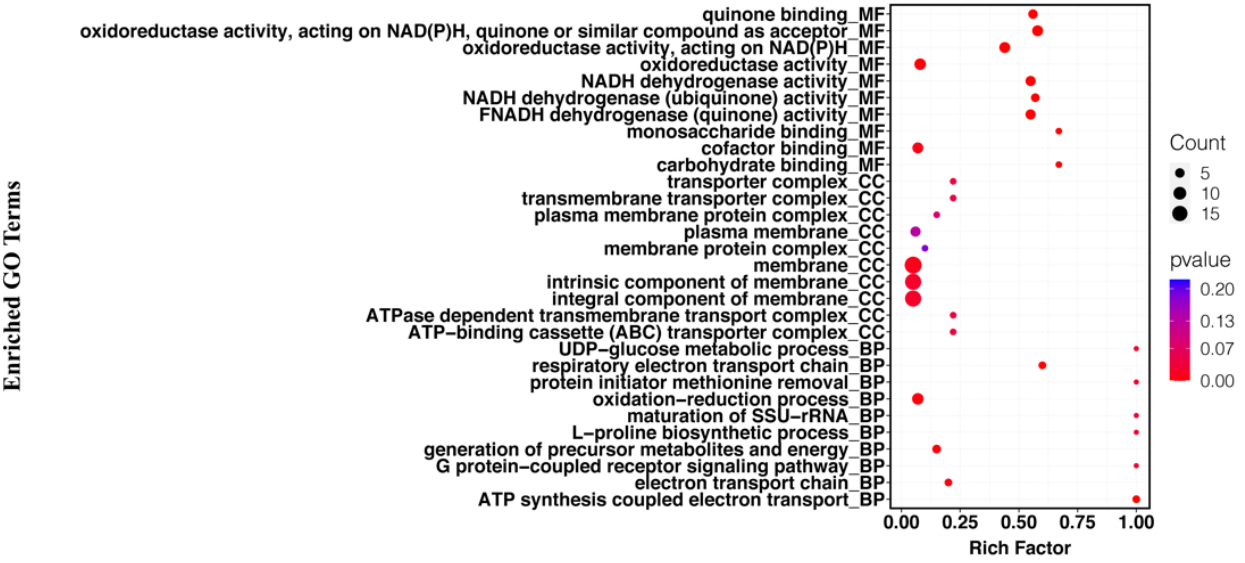

B

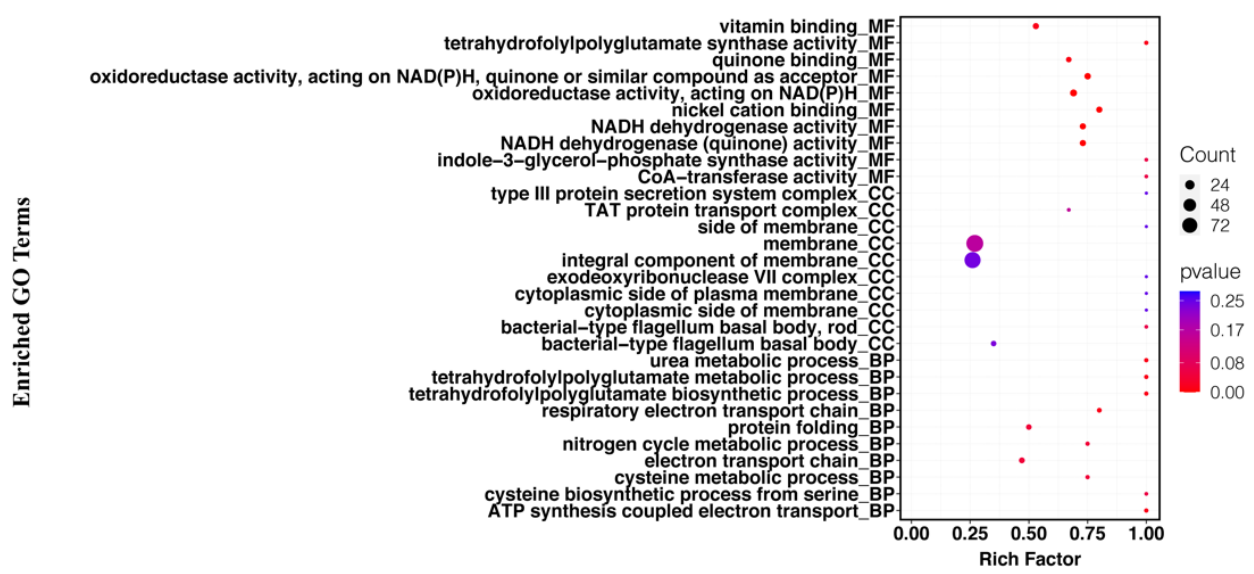

Figure 6. Analysis of GO enrichment for H. pylori 3192 biofilm under different treatments. Top 10 GO terms in the cellular component (CC), molecular component (MF), and biological process (BP) are shown. Rich factor refers to the ratio of DEGs enriched in the pathway to the number of genes annotated. The greater the value is, the higher the enrichment degrees. The size of the solid circle represents the number of DEGs enriched in this pathway, and the color represents the significance of enrichment. FIC group: $1 \times$ FIC of AMX and CLR, CF7 group: $6.25 \%$ CFS of $10^{7} \mathrm{CFU} / \mathrm{mL}$ L. salivarus LN12 in combination with $1 \times$ FIC of AMX and CLR, CF9 group: $6.25 \%$ CFS of $10^{9} \mathrm{CFU} / \mathrm{mL}$ L. salivarus LN12 in combination with $1 \times$ FIC of AMX and CLR. (A) CF7 group vs. FIC group, (B) CF9 group vs. FIC group.

\subsubsection{KEGG Enrichment Analysis}

The distributions of the DEGs of CF7 and CF9 are shown in Figure 8. Compared with the FIC group, the DEGs in CF7 were related to metabolism (carbohydrate metabolism, energy metabolism, lipid metabolism, amino acid metabolism, metabolism of cofactors and vitamins, and biosynthesis of other secondary metabolites), genetic information processing (translation, folding, sorting, and degradation), and environmental information processing (membrane transport, signal transduction). In addition to the above pathways, DEGs were found to be related to metabolism (nucleotide metabolism, metabolism of cofactors and vitamins, metabolism of terpenoids and polyketides, biosynthesis of other secondary metabolites, and xenobiotic biodegradation and metabolism), organismal systems (immune system, environmental adaptation), human diseases (endocrine and metabolic diseases, infectious diseases: bacteria, drug resistance: antimicrobials), genetic information processing (transcription, replication, and repair), and cellular processes (flagellar assembly, biofilm formation, bacterial chemotaxis, quorum sensing) in CF9. Overall, compared with the FIC group, CF9 had more DEGs than CF7, and the number of KEGG pathways enriched in CF9 was greater. The significant pathway differences between the CF7 and CF9 groups 
were mainly reflected in metabolism, genetic information processing, and environmental information processing, especially in flagellar assembly, bacterial chemotaxis, quorum sensing, and antibiotic resistance for CF9.

A

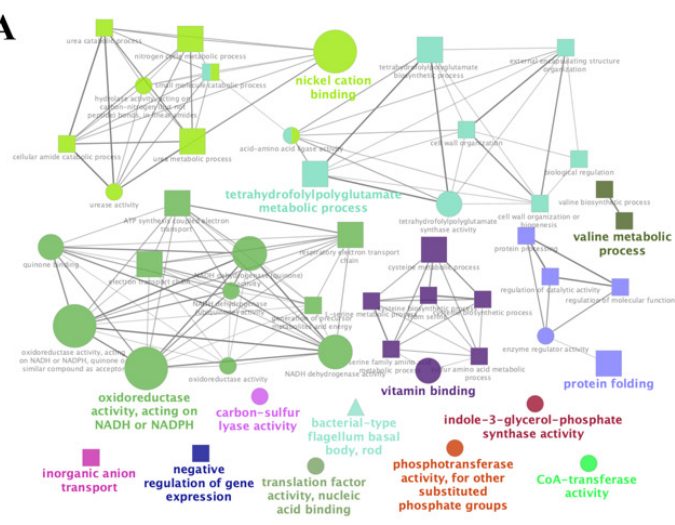

B

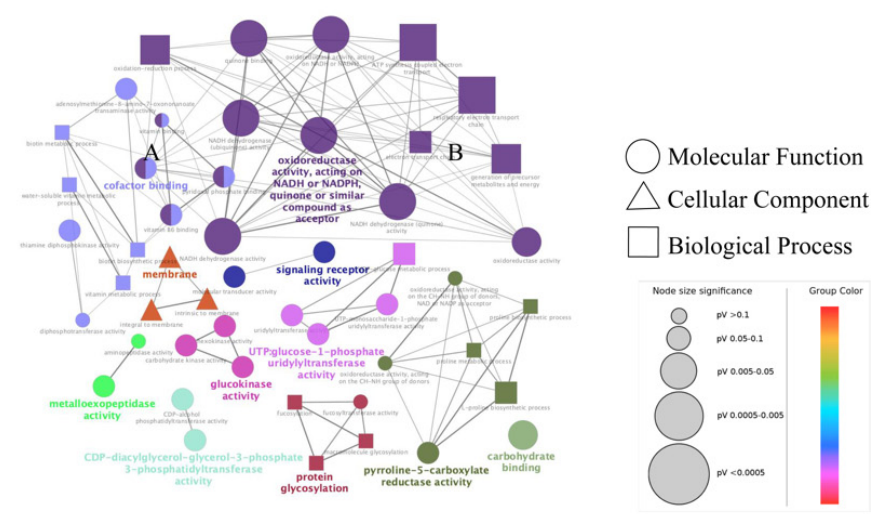

Figure 7. ClueGO analysis for GO terms $(p<0.1)$ in H. pylori 3192 biofilm under different treatments. Each node in the graph represents a GO term, the connection between nodes reflects the correlation between terms, and the color of the nodes reflects the enrichment and classification of the node. Significant GO terms are marked with color. FIC group: $1 \times$ FIC of AMX and CLR, CF7 group: $6.25 \%$ CFS of $10^{7} \mathrm{CFU} / \mathrm{mL}$ L. salivarus LN12 in combination with $1 \times$ FIC of AMX and CLR, CF9 group: $6.25 \%$ CFS of $10^{9} \mathrm{CFU} / \mathrm{mL}$ L. salivarus LN12 in combination with $1 \times$ FIC of AMX and CLR. (A): CF7 group vs. FIC group, (B): CF9 group vs. FIC group.

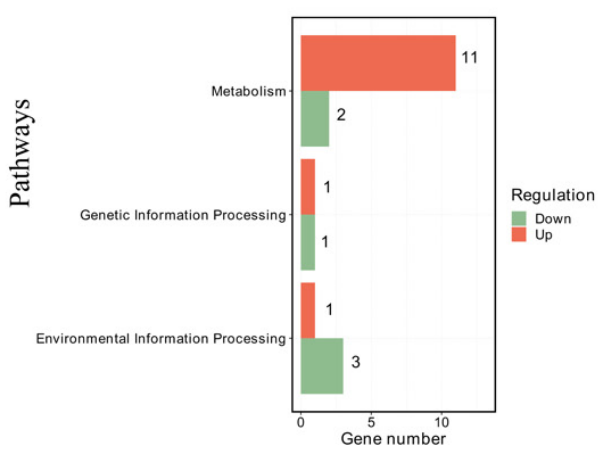

DEGs in KEGG level1 in CF9 group vs FIC group

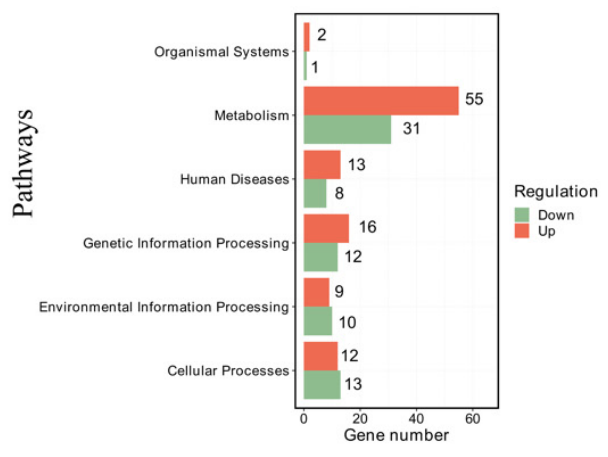

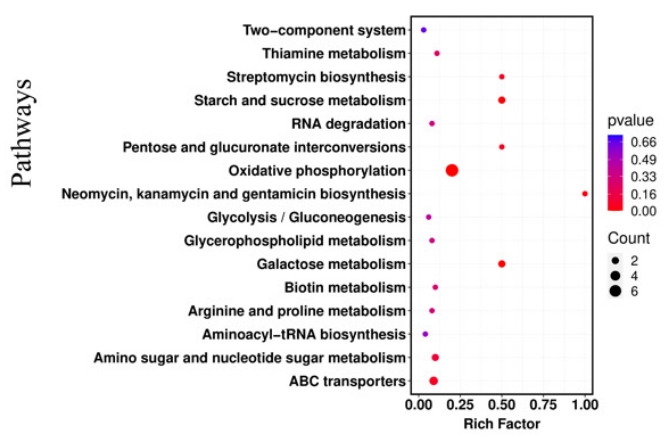

KEGG pathway Enrichment in CF9 group vs FIC group

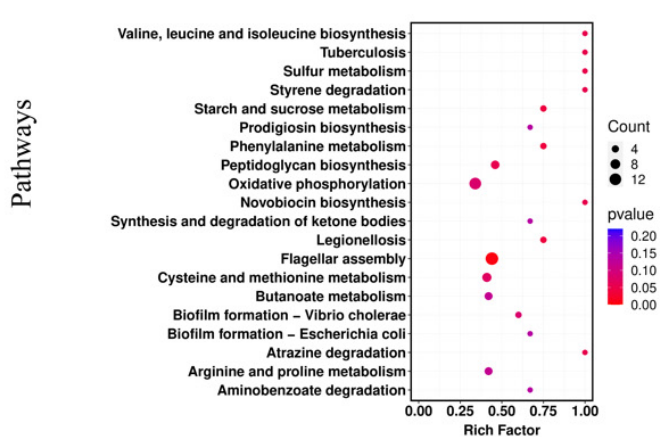

Figure 8. KEGG pathway and enrichment for H. pylori 3192 biofilm under different treatments. For the KEGG pathway database, biological metabolic pathways were divided into cellular processes, environmental information processing, genetic information processing, human diseases, metabolism, 
and biological systems in level 1. Rich factor refers to the ratio of DEGs enriched by the pathway to the number of genes annotated. The greater the factor is, the higher the enrichment degree is. For the enrichment analysis results, the size of the solid circle represents the number of DEGs enriched on this path, and the color represents the significance of enrichment. FIC group: $1 \times$ FIC of AMX and CLR, CF7 group: $6.25 \%$ CFS of $10^{7}$ CFU/mL L. salivarus LN12 in combination with $1 \times$ FIC of AMX and CLR, CF9 group: $6.25 \%$ CFS of $10^{9} \mathrm{CFU} / \mathrm{mL}$ L. salivarus LN12 in combination with $1 \times$ FIC of AMX and CLR.

Compared with the FIC group, the number of DEGs related to metabolism was the highest in CF7 and CF9. In total, 11 genes in the CF7 group were upregulated and 2 were downregulated while 55 genes in the CF9 group were upregulated and 31 were downregulated. The KEGG pathways shared by the CF7 and CF9 groups were glycolysis/gluconeogenesis, pentose and glucuronate interconversions, galactose metabolism, starch and sucrose metabolism, amino sugar and nucleotide sugar metabolism, oxidative phosphorylation, glycerophospholipid metabolism, arginine and proline metabolism, thiamine metabolism, biotin metabolism, streptomycin biosynthesis, neomycin, kanamycin, and gentamicin biosynthesis. CF9 also had DEGs related to the citrate cycle (TCA cycle), butanoate metabolism, carbon fixation pathways in prokaryotes, fatty acid biosynthesis, purine metabolism, and pyrimidine metabolism. The number of DEGs in oxidative phosphorylation in the CF7 and CF9 group was the largest, but the number in CF9 was greater than that in CF7, and the genes encoding Helicobacter pylori NADH-quinone oxidoreductase subunits M, J, L, NuoK, NuoH, NuoI, and NuoN were upregulated in both CF7 and CF9: 2.9-4.3-fold in CF7 and 5.7-8.3-fold in CF9. The genes encoding Helicobacter pylori NADHquinone oxidoreductase subunits $\mathrm{G}$ and $\mathrm{D}$ and the fumarate reductase iron-sulfur subunit were upregulated 2.2-3.3-fold in CF9. The gene encoding Helicobacter pylori ATP F0F1 synthase subunit B was downregulated 0.4-fold in CF9. In starch and sucrose metabolism, the genes encoding UTP-glucose-1-phosphate uridylyltransferase GalU and glucokinase were upregulated 2.3-2.7-fold in CF7 and 3.2-4.4-fold in CF9, and the glucokinase gene was upregulated 3.6-fold in CF9. In genetic information processing, the KEGG pathways shared by CF7 and CF9 were aminoacyl-tRNA biosynthesis and RNA degradation. On this basis, CF9 contains 10 other pathways, including ribosome and protein export. The gene encoding Helicobacter pylori Asp-tRNA(Asn)/Glu-tRNA(Gln) amidotransferase subunit GatC was upregulated 2.7-fold in CF7; the genes encoding Helicobacter pylori Asp-tRNA(Asn)/GlutRNA(Gln) amidotransferase subunits GatC and GatB, phenylalanine-tRNA ligase subunit beta, and proline-tRNA ligase were upregulated 2.15-9.0-fold in CF9; and the argS gene was downregulated 0.44-fold in CF9.

For environmental information processing, the CF7 and CF9 group shared DEGs in $\mathrm{ABC}$ transporters and the two-component system. For $\mathrm{ABC}$ transporters, the genes encoding LPS export ABC transporter ATP-binding protein, cysteine ABC transporter permease, and transporter substrate-binding domain-containing protein were downregulated 0.44-0.47-fold in CF7, and the genes encoding ABC transporter substrate-binding protein, ABC transporter ATP-binding protein, ATP-binding cassette domain-containing protein, and osmoprotection protein were downregulated 0.37-0.48-fold in CF9. In the 2-component system, the genes encoding Helicobacter pylori TIGR00366 family proteins were upregulated 2.2-fold in CF7 and CF9; the genes encoding RNA polymerase sigma factor fliA and czcA were upregulated 2.2-3.8-fold in CF9; and the genes encoding McpB, flagellin, and flagellar motor stator protein were downregulated 0.20-0.44-fold in CF9. Compared with the CF7 group, the same gene in the same KEGG pathway was upregulated by a higher multiple in CF9, and the number of pathways was greater in CF9.

Flagellar assembly, quorum sensing, bacterial chemotaxis, and antibiotic resistance are all related to $H$. pylori biofilms. Compared with the FIC group, DEGs in the above pathways were not found in CF7 but were found in CF9. In total, 14 DEGs were related to flagellar assembly: the genes encoding flagellar motor switch proteins fliY and fliM and the RNA polymerase sigma factor fliA were upregulated 2.6-3.8-fold while the genes encoding 
flagellar hook protein flgE, flagellar basal body rod protein flgC, and flagellin were downregulated $0.15-0.45$-fold. In total, 4 DEGs were related to quorum sensing: the genes encoding anthranilate synthase, membrane protein insertase YidC, and S-ribosylhomocysteinase were upregulated 2.0-3.0-fold, and the gene encoding preprotein translocase subunit SecG was downregulated 0.44 -fold. In total, 4 DEGs were related to bacterial chemotaxis: the genes encoding flagellar motor switch proteins fliY and fliM were upregulated 2.5-3.0-fold, and the genes encoding HAMP domain-containing protein, $\mathrm{ABC}$ transporter substratebinding protein, flagellar motor protein $\mathrm{MotB}$, and flagellar motor stator protein were downregulated 0.38-0.44-fold. In total, 7 DEGs were related to biofilm formation: the genes encoding RNA polymerase sigma factor fliA, S-ribosylhomocysteinase, and anthranilate synthase were upregulated 2.0-3.8-fold, and the gene encoding serine O-acetyltransferase was downregulated 0.44 -fold. In total, 5 DEGs were related to antimicrobial drug resistance: the genes encoding copper-translocating P-type ATPase CopA and aminotransferase class I/II-fold pyridoxal phosphate-dependent enzyme were upregulated 2.2-2.8-fold while the genes encoding penicillin-binding protein (pbp-1a), D-alanine ligase, and $\operatorname{mur} G$ were downregulated 0.22-0.41-fold. Overall, the CF9 group was more active than the CF7 group in H. pylori biofilms, especially in flagellar assembly, bacterial chemotaxis, quorum sensing, and antibiotic resistance.

The top KEGG pathways with $p$ value $<0.1$ are shown in Supplementary Table S2. Compared with the FIC group, the pathways shared by the CF7 and CF9 groups were oxidative phosphorylation and starch and sucrose metabolism. The different pathways included carbohydrate metabolism, biosynthesis of other secondary metabolites, membrane transport in CF7, and amino acid metabolism, energy metabolism, biosynthesis of other secondary metabolites, xenobiotic biodegradation and metabolism, cell motility, and cellular community-prokaryotes in CF9.

Compared with the FIC group, the top 20 genes with the most upregulated and downregulated levels in CF7 and CF9 are listed in Supplementary Table S3. The most upregulated gene in CF7 was 1_orf01846, at 4.26-fold, which was related to Helicobacter pylori $\mathrm{NADH}$-quinone oxidoreductase subunit $\mathrm{M}$, and other genes related to NADH-quinone oxidoreductase subunit genes were upregulated almost 3-fold. The most upregulated gene in CF9 was 1_orf00834, with a 12.10-fold increase. Other genes related to the NADH-quinone oxidoreductase subunit were upregulated almost 5.67-fold. Overall, the most upregulated genes in both the CF7 and CF9 groups were related to NADH-quinone oxidoreductase, and the results are in accordance with KEGG and GO enrichment, all of which showed that the oxidative phosphorylation pathway was active. The most downregulated gene in CF7 was 1_orf01871, at 0.63-fold, which was related to thiamine diphosphokinase. The most downregulated gene in CF9 was adenosylmethionine-8-amino-7-oxononanoate transaminase, which was downregulated 0.13 -fold. Interestingly, some flagellum-related genes were significantly downregulated. The results are in accordance with KEGG enrichment, and all showed that pathways related to flagellar assembly were significant in the CF9 group.

\subsubsection{RT-qPCR Validation}

To validate the RNA-Seq, eight DEGs for CF7 (nuoI, nuoN, membrane, flgK, flgL, motB, $f l g B, f l g C$ ) and eight DEGs (nuoI, nuoN, rbfA, dnaK, ure A, fliH, sabA, vacA) for CF9 were chosen for RT-qPCR. The FIC group was used as the control group, and the results are shown in Figure 9. The change trend of the above genes was consistent in RT-qPCR and RNA-Seq. 

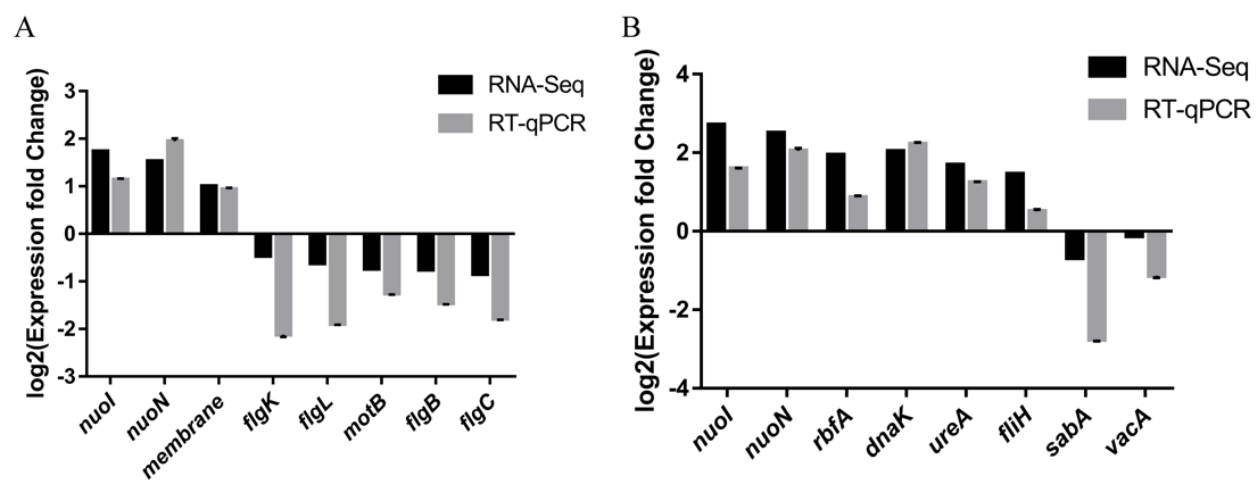

Figure 9. Validation of RNA-Seq by RT-qPCR on selected DEGs. FIC group: $1 \times$ FIC of AMX and CLR, CF7 group: $6.25 \%$ CFS of $10^{7} \mathrm{CFU} / \mathrm{mL}$ L. salivarus LN12 in combination with $1 \times$ FIC of AMX and CLR, CF9 group: $6.25 \%$ CFS of $10^{9} \mathrm{CFU} / \mathrm{mL}$ L. salivarus LN12 in combination with $1 \times$ FIC of AMX and CLR. (A): CF7 vs. FIC, (B): CF9 vs. FIC.

\section{Discussion}

Probiotics have been clinically proven to affect the treatment of $H$. pylori, and clinicians recommend the combination of probiotics and antibiotics, but there is no unified standard regarding the strains, dose, timing, and course of treatment of probiotics. A meta-analysis showed that L. plantarum and P. acidilactici were used at a dose of $1 \times 10^{9} \mathrm{CFU}$ for 10 days, L. rhamnosus GG at a dose of $1.2 \times 10^{10} \mathrm{CFU}$ for 14 days, and L. reuteri DSM17938 and L. reuteri ATCC PTA 6475 at a dose of $2 \times 10^{8}$ CFU for 13 weeks [18]. Our previous research found that the combination of L. salivarus LN12 CFS with AMX and CLR had stronger destructive effects on $H$. pylori biofilms in vitro, but no study has explored whether the combination of CFSs of different doses of $L$. salivarus LN12 with AMX and CLR has different effects on $H$. pylori biofilms in vitro, especially on the transcriptome [23]. Therefore, we evaluated the effects of CFSs of high- and low-dose L. salivarus LN12 combined with AMX and CLR on $H$. pylori biofilms in terms of physiological indices and transcriptomes.

$H$. pylori biofilms are related to culture conditions, culture systems, and strains in vitro. Biofilms may effectively protect $H$. pylori from antibacterial agents and the immune system [5]. There are two main methods for forming $H$. pylori biofilms in vitro: biofilms can be formed at the gas-liquid interface in liquid culture or grown on nitrocellulose (NC) membranes attached to blood agar plates $[19,23,25,26]$. H. pylori biofilms were previously used to screen some natural products with anti- $H$. pylori effects. The evaluation indices were the biofilm biomass, the bacterial survival rate in biofilm, and the biofilm structure [27-29]. The results of our previous study showed that the MIC of $10^{9} \mathrm{CFU} / \mathrm{mL}$ L. salivarus LN12 CFS to H. pylori 3192 was $12.5 \%$, and that CFS of $10^{9} \mathrm{CFU} / \mathrm{mL}$ L. salivarus LN12 can enhance the destructive effects of AMX and CLR on H. pylori 3192 biofilm. The effects of CFS alone were verified, and the results indicated that some acids and antibacterial proteins in LN12 CFS had a killing effect on $H$. pylori 3192 biofilm. CFS did not contain hydrogen peroxide or contained too low a concentration of hydrogen peroxide, and the metabolites in CFS that played a role were thermally stable; these factors may have all had effects [23]. For better observation, $1 / 2$ MIC (6.25\%) of L. salivarus LN12 CFS was combined with AMX and CLR. To compare the effects of different doses of $L$. salivarus LN12 CFS combined with AMX and CLR, $6.25 \%$ CFS of $10^{5} \mathrm{CFU} / \mathrm{mL}, 10^{7} \mathrm{CFU} / \mathrm{mL}$, and $10^{9} \mathrm{CFU} / \mathrm{mL}$ L. salivarus $\mathrm{LN} 12$ were used with AMX and CLR, named the CF5, CF7, and CF9 groups, respectively [23]. The results showed that the higher the dose of L. salivarus LN12 combined with AMX and CLR, the more significant the destruction of the biofilm biomass, survival rate, and structure, which may suggest that although probiotics have therapeutic effects on $H$. pylori, a certain dose is necessary to exert a significant therapeutic effect, and the effect of a low dose may be nonsignificant.

At present, there are few studies on the transcriptome of $H$. pylori biofilms. Hathrougbi compared the transcriptome of $H$. pylori SS1 in planktonic and biofilm forms at the gas- 
liquid interface [6]. H. pylori biofilms are difficult to analyze by RNA-Seq because when extracting RNA after different treatments, too long a treatment time or errors when operating the technology can cause RNA degradation. RNA-Seq requires high purity and integrity of the RNA. This experiment found that RNA from H. pylori 3192 biofilms subjected to different treatments needs to be extracted immediately, and quick freezing by liquid nitrogen leads to RNA degradation. The H. pylori biofilm contains sugars, proteins, etc., and we ground and homogenized the sample before extracting the RNA, which greatly increased the total amount of RNA. In this study, the transcriptome of $H$. pylori biofilms formed at the gas-liquid interface and after being subjected to different treatments, it was studied for the first time to provide a reference method for the transcriptome of $H$. pylori biofilms [23].

Compared with the FIC group, KEGG and GO enrichment analysis showed that a large number of DEGs in CF9 and CF7 were related to the oxidative phosphorylation process. H. pylori, an obligate microaerophilic bacterium, contains two NADH-quinone oxidoreductases in the respiratory chain: proton-translating NADH-quinone oxidoreductase (NDH-1 or complex I) and NADH-quinone reductase (NDH-2). NDH-1 is located on the inner membrane of mitochondria and catalyzes the transfer of electrons from NADH to the quinone pool through a series of redox centers [30,31]. When organisms are under stress, oxidative phosphorylation, carbon assimilation, and the tricarboxylic acid cycle are involved in antitoxicity processes. It was found that by increasing the time of exposure to sodium fluoride (NAF), the damage to silkworm testes became more serious, indicating that $\mathrm{NaF}$ stress affected the NADH respiratory chain of the mitochondrial electron transport chain, increased the activity of related enzyme complexes, and significantly increased the content of intracellular ROS, resulting in an intracellular oxidative stress response [32]. NADH is the main cofactor required to reduce toxic compounds to low toxic compounds. The level of NADH/NAD+ is related to oxidative stress. The response of Salmonella typhimurium to vanillin in apple juice was studied by transcriptome analysis, and it was found that some NADH-related genes were upregulated (nио $A, n и о B, n u о K)$, which increased the oxidative stress response [33]. When $H$. pylori 3192 biofilms were treated with the CFS of high-dose L. salivarus LN12 combined with AMX and CLR, the oxidative phosphorylation of biofilms was more active, and the increase in the expression of nuoK, nuoH, nuoI, nuoN, and other subunit genes of NDH-1 in CF9 was significantly higher than that in CF7. It was speculated that CF9 could cause stronger damage to H. pylori biofilm cells, causing H. pylori 3192 to enter a stronger stress state with increased NADH-related gene expression, and a stronger oxidative stress response to help "detoxify". Physiological indicators showed that CF9 had stronger effects on the biomass, survival rate, and structure of H. pylori 3192 biofilms.

Compared with the FIC group, some DEGs occurred in flagellar assembly, quorum sensing, bacterial chemotaxis, biofilm formation, and antimicrobial drug resistance in the CF9 group but not in the CF7 group. The gene regulation process of $H$. pylori biofilms has not been fully explained [34]. Hathrougbi compared the biofilm and planktonic forms of SS1 through transcriptome analysis and found that the genes related to flagellum formation (e.g., $f l g L, f l g K, f l g B, f l g E$, and $f l i K)$ were significantly upregulated and might be related to biofilm formation, and $\Delta$ motB and $\Delta$ flimA were significantly downregulated in biofilms [6]. We found that after treatment with the CFS of high-dose L. salivarus LN12 and AMX and $C L R, f l g K, f l g L, f l g E, f l g B$, and $m o t B$ were downregulated in $H$. pylori 3192 biofilms. The strong destructive effects of CF9 on the biomass, survival rate, and structure of $H$. pylori 3192 biofilms may be related to the downregulation of these genes.

Bacteria can regulate biofilms through quorum sensing communication. H. pylori has only one known quorum sensing system, automatic inducer 2 (AI-2), synthesized by the LuxS gene. H. pylori senses AI-2 as negative chemotaxis through the chemical receptor $\mathrm{TlpB}$, and chemorepulsion by AI-2 is the decisive factor regarding the spatial organization and diffusion of $H$. pylori biofilms [34,35]. Early colonization of Arabidopsis roots by Bacillus subtilis is mediated by the characteristic chemical receptors $\mathrm{McpB}$ and $\mathrm{McpC}$ and TlpC, and $\Delta m c p b$ can significantly reduce this early colonization [36]. We found that the $m c p b$ 
gene was significantly downregulated after H. pylori 3192 biofilms were treated with CF9. We speculate that the stronger destructive effect of CF9 on H. pylori 3192 biofilms may be related to the downregulation of the $m c p b$ gene.

Studies have shown that subinhibitory concentrations (sub-MICs) of antibiotics increase bacterial tolerance to antibiotics and the risk of drug resistance. The FIC concentrations of AMX and CLR used in this study were lower than MIC, which raised the question of why DEGs related to drug resistance did not appear in the CF7 group distribution [37,38]. When we explored the effects of CFSs of different doses of L. salivarus LN12 in combination with antibiotics, DEGs related to drug resistance did not appear in the CF7 group when the FIC group was used as the control but did appear in the CF7 group when the BB2 group was used as the control. Fourteen DEGs were validated by qPCR: nuoI and nuoN were related to NDH-1 activity; $f \lg K, f \lg L, f l g B, f l g C, \operatorname{mot} B$, and $f l i H$ were related to flagella; $r b f A$ was related to translation and regulation; $d n a K$ was related to heat shock; and $s a b A$ and $v a c A$ were related to virulence [39-47]. In our previous study, the vacA gene was downregulated significantly when L. salivarus LN12 CFS was combined with AMX and CLR, which was confirmed in this study [23].

Yang found that probiotics did not show a dose effect on Clostridioides difficile (CD) in vitro through transcriptome analysis. High-dose Bifidobacterium breve YH68 may have failed because it induced CD to produce more toxins and spores, and low-dose YH68 may have failed because it stimulated CD to exhibit stronger drug resistance [48]. In this study, physiological indices and transcriptome results both showed that the effects of high-dose L. salivarus LN12 CFS combined with antibiotics on H. pylori 3192 biofilm were greater than that of low-dose L. salivarus LN12 CFS. We suggest that high-dose L. salivarus LN12 should be used in the clinical adjuvant treatment of $H$. pylori, and the effects of low-dose L. salivarus LN12 are not obvious. We propose the concept of a "threshold", where the effects of probiotic may be more obvious when the dose is greater than the "threshold". We also need to recognize that the dose effects of L. salivarus LN12 may be related to strain specificity. To facilitate the performance of the experiment, our laboratory used CFS instead of the L. salivarus LN12 strain.

\section{Materials and Methods}

\subsection{Strains and Culture Conditions}

H. pylori clinical isolate 3192 was provided by Renji Hospital of Shanghai Jiaotong University (Shanghai, China), and Lactobacillus salivarius (LN12) was provided by Jiaxing Innocul-Probiotics Co., Ltd. (Jiaxing, China). H. pylori was cultured on Columbia blood agar plates containing 5\% sterile defibrinated blood or in Brinell broth medium containing $10 \%$ Gibco fetal bovine serum (BB10) for proliferation. $H$. pylori biofilms were cultured in Brinell broth medium containing $2 \%$ fetal bovine serum (BB2), all at $37^{\circ} \mathrm{C}$ in a microaerobic environment (MGC, Tokyo, Japan) for 48-72 h, and the speed of liquid culture was set to $100 \mathrm{rpm}$. With reference to Jin and Ji, modified MRS medium was used for L. salivarus LN12 at $37^{\circ} \mathrm{C}$ for $24-48 \mathrm{~h}[19,23]$.

\subsection{H. pylori Biofilm Culture}

The method of culturing H. pylori biofilm was reported by Jin: after activation for 2 generations, H. pylori was cultured in a 12-well sterile cell culture plate with a $20 \mathrm{~mm} \times 20 \mathrm{~mm}$ borosilicate coverslip (Matsunami Glass, Tokyo, Japan) and $2 \mathrm{~mL}$ of BB2 in each well, and the initial concentration of $H$. pylori 3192 in each well was no less than $10^{7} \mathrm{CFU} / \mathrm{mL}$. The speed was set at $80 \mathrm{rpm}$, and $H$. pylori biofilm was formed at the gas-liquid interface at $37^{\circ} \mathrm{C}$ in microaerobic culture for 4 days [23].

4.3. Preparation of the Combination of Lactobacillus Salivarius LN12 Cell-Free Supernatant (CFS) and Antibiotics

According to our previous study, the FIC concentrations of AMX and CLR for H. pylori 3192 were $2 \times \mathrm{MIC}_{\mathrm{CLR}}$ and $0.125 \times \mathrm{MIC}_{\mathrm{AMX}}, \mathrm{MIC}_{\mathrm{CLR}}$ was $0.016 \mathrm{mg} / \mathrm{L}, \mathrm{MIC}_{\mathrm{AMX}}$ was 
$0.016 \mathrm{mg} / \mathrm{L}$, and the MIC of L. salivarus LN12 (109 CFU/mL) CFS on H. pylori 3192 was $12.5 \%$ [23]. When AMX and CLR were used in combination with L. salivarus LN12 CFS, the concentration was set to $1 \times$ FIC and $1 / 2 \times$ MIC of L. salivarus LN12 CFS (6.25\%) [23]. To compare the effects of the CFS from different doses of L. salivarus LN12 with antibiotics, CFSs from different doses of L. salivarus LN12 were all diluted to $6.25 \%$ as follows: after activation for 2 generations, L. salivarus LN12 was cultured in mMRS to the logarithmic phase at $37^{\circ} \mathrm{C}$; diluted with BB2 to concentrations of $10^{9}, 10^{7}$, and $10^{5} \mathrm{CFU} / \mathrm{mL}$; and then centrifuged at $7600 \mathrm{rpm}$ and $4{ }^{\circ} \mathrm{C}$ for $10 \mathrm{~min}$. The CFS was filtered with a $0.22 \mu \mathrm{m}$ sterile filter membrane and diluted to $6.25 \%$ with BB2, then finally combined with $1 \times$ FIC of AMX and CLR. According to the dose of L. salivarus LN12, the groups were named as follows: CF9 group $\left(6.25 \%\right.$ CFS of $10^{9} \mathrm{CFU} / \mathrm{mL}$ L. salivarus LN12 in combination with $1 \times$ FIC of AMX and CLR), CF7 group (6.25\% CFS of $10^{7}$ CFU / mL L. salivarus LN12 in combination with $1 \times$ FIC of AMX and CLR), CF5 group (6.25\% CFS of $10^{5} \mathrm{CFU} / \mathrm{mL}$ L. salivarus LN12 in combination with $1 \times$ FIC of AMX and CLR), and FIC group $\left(2 \times \mathrm{MIC}_{\mathrm{CLR}}\right.$ and $\left.0.125 \times \mathrm{MIC}_{\mathrm{AMX}}\right), \mathrm{BB} 2$ was set as the control.

\subsection{Effects of Different Treatments on the Viability of H. pylori Biofilms}

The viability of $H$. pylori biofilms after different treatments was determined according to the CFU counting method on plates with reference to Jin: 4-day H. pylori 3192 biofilms on coverslips cultured in a 12-well cell culture plate were washed twice with PBS and separately placed in $2 \mathrm{~mL}$ of CF9, CF7, CF5, FIC of AMX and CLR, and BB2 as the control at $80 \mathrm{rpm}$ and $12 \mathrm{~h}$ under microaerobic conditions. After washing twice with PBS, the biofilm was resuspended in $1 \mathrm{~mL}$ of PBS with a cell scraper. CFUs in the biofilm were counted on Columbia agar plates containing $5 \%$ sterile defibrinated sheep blood under microaerobic conditions after $72 \mathrm{~h}[23]$.

\subsection{Effects of Different Treatments on the Biomass of H. pylori Biofilms}

The H. pylori 3192 biofilms were subjected to different treatments as described above. After washing twice with PBS, the biofilm on the coverslips was stained with $1 \%$ crystal violet for $20 \mathrm{~min}$ and then resuspended in $400 \mu \mathrm{L}$ of $95 \%$ ethanol. The absorbance at a wavelength of $595 \mathrm{~nm}$ was measured by a spectrophotometer [23].

\subsection{Effects of Different Treatments on the Structure of H. pylori Biofilm}

H. pylori 3192 biofilms on coverslips were observed under scanning electron microscopy (SEM) and confocal laser scanning microscopy (CLSM) according to Jin [23]. After being subjected to different treatments as described above, for SEM observation, after fixation overnight with 50\% glutaraldehyde and dehydration with 50-100\% gradient ethanol, the biofilm was dried at the critical point, then sprayed with gold (Leica, Wetzlar, Germany) and observed under SEM (S-4800, Hitachi, Tokyo, Japan). For CLSM, the biofilm was placed in a 1:1 mixture of SYTO 9 and propidium iodide (Invitrogen, Carlsbad, CA, USA) for $20 \mathrm{~min}$ in the dark and then observed under CLSM (Nikon, Tokyo, Japan) using an oil objective. The excitation wavelengths were 488 and $560 \mathrm{~nm}$, and the Z-axis acquisition signal step length was $0.1 \mu \mathrm{m}$. Nikon Ni-E A1 HD25 special software was used to analyze the image [23].

\subsection{Preparation of RNA-Seq Sequencing Library}

First, 4-day H. pylori 3192 biofilms cultured in 12-well cell culture plates were washed twice with PBS and separately placed in $2 \mathrm{~mL}$ of CF9, CF7, FIC of AMX and CLR, or BB2 (used as a control) at $80 \mathrm{rpm}$ and $37^{\circ} \mathrm{C}$ for $12 \mathrm{~h}$ under microaerobic conditions. Four independent biological samples were used for every group. H. pylori 3192 biofilms on the plate wall were collected with a cell scraper, and the extraction method to obtain biofilm RNA was slightly adjusted on the basis of Wang. Homogenate grinding at low temperature was carried out before extraction [49]. RNA was extracted with a TransZol Up Plus RNA Kit (TransGen Biotech, Beijing, China), and RNA quality and quantity were evaluated using 
1\% agarose gel electrophoresis, Qubit 3.0 (Thermo Fisher Scientific, Bannockburn, MA, USA), Nanodrop One (Thermo Fisher Scientific, Bannockburn, MA, USA), and Agilent 4200 (Agilent Technologies, Waldbron, Germany). An ALFA-SEQ rRNA Depletion Kit was used to remove ribosomal RNA. The library was constructed using the NEB Next UltraTM Directional RNA Library Prep Kit for Illumina (New England Biolabs, Ipswich, MA, USA), qualified libraries were generated using the Illumina NovaSeq6000 platform, and $150 \mathrm{bp}$ paired-end reads were generated.

\subsection{Bioinformatics Analysis}

Clean reads were obtained by quality control of raw reads with Trimmomatic (v.0.36), ribosomal sequences were removed by Bowtie2 software, and reads were mapped to the annotated reference Helicobacter pylori strain 3192 chromosome complete genome in NCBI (GenBank: CP086760). The sequencing quality was judged by RSeQC software and a homemade script, and the expression was counted by RSEM software. The expression level was measured in fragments per kilobase per million reads (FPKM). Differential gene analysis was performed on multiple samples using read counts, differentially expressed genes (DEGs) were analyzed with edgeR (v3.16.5) with default screening conditions of FDR $\leq 0.05$ and $\mid \log 2$ (fold change) $\mid \geq 1$, and the correction method of multiple inspection was BH (fdr correction with Ben-jamini/Hochberg). Principal component analysis (PCA), Venn diagrams, and heat maps were used to analyze the relationships between group samples. Cluster profiler (v3.4.4) was used to analyze the functions of the DEGs to determine the roles of these genes in cells and the metabolic pathways involved. The annotation databases used were Gene Ontology (GO) and Kyoto Encyclopedia of Genes and Genomes (KEGG). GO terms with $p$ value $<0.1$ were selected and further analyzed with the ClueGO plugin in Cytoscape (3.9.0), and Helicobacter pylori 26,695 was used as a loading marker [48,50].

\subsection{RT-qPCR Validation}

Fourteen DEGs were selected to validate the RNA-Seq results with RT-qPCR, and RNA from the control group, FIC group, $\mathrm{CF} 7$ group, and $\mathrm{CF} 9$ group was reverse transcribed with the PrimeScriptTM RT Reagent Kit with gDNA Eraser No. RR047A (Perfect Real Time) (Takara, Kyoto, Japan). QPCR was performed using TaKaRa TB Green Premix Ex Taq ${ }^{\mathrm{TM}}$ II (Tli RNaseH Plus) (Code No. RR820A) (Takara, Kyoto, Japan) on a quantitative PCR apparatus (Analytikjena, Jena, Germany). The primers used are listed in Supplementary Table S4, and the results were analyzed using the $2^{-\Delta \Delta C T}$ method. The $16 \mathrm{~S}$ rRNA gene was used as an internal reference.

\subsection{Data Analysis and Availability}

All experimental data were expressed as the mean \pm standard deviation $(n=3)$, and statistical analysis was performed by one-way analysis of variance (ANOVA) and Tukey's multiple comparison test using OriginPro Learning Edition (OriginLab, Northampton, MA, USA). A $p$ value $\leq 0.05$ was considered statistically significant.

\section{Conclusions}

In this study, H. pylori 3192 biofilm was treated with CFSs of high- and low-dose L. salivarus LN12 combined with AMX and CLR for the first time in vitro. The changes in biofilms were characterized by physiological indices and transcriptomes. Compared with that of low-dose L. salivarus LN12, the CFS of high-dose L. salivarus LN12 combined with AMX and CLR had more significant destructive effects on the H. pylori 3192 biofilm biomass, survival rate and biofilm structure. The transcriptome results showed that compared with the AMX and CLR treatment group, the largest number of DEGs for treatment with high- and low-dose L. salivarus LN12 CFSs combined with AMX and CLR were related to oxidative phosphorylation; more DEGs were related to oxidative phosphorylation for the high-dose L. salivarus LN12 CFS; and in particular, DEGs related to flagellar assembly, bacterial chemotaxis, quorum sensing, antibiotic resistance, and H. pylori biofilm formation appeared in the 
high-dose group. Compared with the low-dose L. salivarus LN12 combination, H. pylori 3192 may produce a stronger oxidative stress response by increasing NADH-related genes and downregulating flagellar assembly-related and quorum sensing-related receptor genes to cope with the stronger destructive effects on the biofilms of high-dose L. salivarus LN12 in combination with AMX and CLR. This study suggests that when L. salivarus LN12 is combined with AMX and CLR for the treatment of $H$. pylori biofilms, the therapeutic effects of high-dose probiotics may be better than those of low-dose probiotics, and the effects may be more obvious when the dosage of probiotics is higher than a certain "threshold".

Supplementary Materials: The following supporting information can be downloaded at: https: / / www.mdpi.com/article/10.3390/antibiotics11020262/s1, Supplementary Figure S1: Sample expression pattern, Supplementary Figure S2: Volcano plot, Supplementary Table S1: RNA sample quality results, Supplementary Table S2: Top KEGG pathway $(p<0.1)$ in CF7 and CF9, Supplementary Table S3: The top 20 up- and downregulated genes in CF7 and CF9, Supplementary Table S4: Real-time PCR primers.

Author Contributions: Conceptualization, F.J.; Funding acquisition, H.Y.; Methodology, F.J.; Project administration, H.Y.; Resources, H.Y.; Supervision, H.Y.; Visualization, F.J.; Writing-original draft, F.J.; Writing-review and editing, H.Y. All authors have read and agreed to the published version of the manuscript.

Funding: This work was funded by Shanghai Industry-University Joint Research Program, grant numbers HUCXY-2016-010.

Data Availability Statement: Raw data are publicly available at Sequence Reads Archive with sample name SAMN23509605 (Control group), SAMN23509606 (FIC group), SAMN23509607 (CF7 group), SAMN23509608 (CF9 group).

Conflicts of Interest: The authors declare no conflict of interest.

\section{References}

1. Chmiela, M.; Kupcinskas, J. Review: Pathogenesis of Helicobacter pylori infection. Helicobacter 2019, 24 (Suppl. 1), e12638. [CrossRef] [PubMed]

2. Hu, Y.; Zhu, Y.; Lu, N.H. Recent progress in Helicobacter pylori treatment. Chin. Med. J.-Peking 2020, 133, 335-343. [CrossRef] [PubMed]

3. Liu, Q.; Meng, X.; Li, Y.; Zhao, C.N.; Tang, G.Y.; Li, S.; Gan, R.Y.; Li, H.B. Natural Products for the Prevention and Management of Helicobacter pylori Infection. Compr. Rev. Food Sci. Food Saf. 2018, 17, 937-952. [CrossRef] [PubMed]

4. Salama, N.R.; Hartung, M.L.; Müller, A. Life in the human stomach persistence strategies of the bacterial pathogen Helicobacter pylori. Nat. Rev. 2013, 11, 385-398. [CrossRef] [PubMed]

5. Hathroubi, S.; Servetas, S.L.; Windham, I.; Merrell, D.S.; Ottemann, K.M. Helicobacter pylori Biofilm Formation and Its Potential Role in Pathogenesis. Microbiol. Mol. Biol. Rev. 2018, 82, 1-18. [CrossRef]

6. Hathroubi, S.; Zerebinski, J.; Ottemann, K.M. Helicobacter pylori Biofilm Involves a Multigene Stress-Biased Response, Including a Structural Role for Flagella. mBio 2018, 9, e01973-18. [CrossRef]

7. Xia, M.; Chen, H.; Liu, S. The synergy of resveratrol and alcohol against Helicobacter pylori and underlying anti-Helicobacter pylori mechanism of resveratrol. J. Appl. Microbiol. 2020, 128, 1179-1190. [CrossRef]

8. Hathroubi, S.; Hu, S.; Ottemann, K.M. Genetic requirements and transcriptomics of Helicobacter pylori biofilm formation on abiotic and biotic surfaces. NPJ Biofilms Microbioms 2020, 6, 56. [CrossRef]

9. Zhao, Y.; Cai, Y.; Chen, Z.; Li, H.; Xu, Z.; Li, W.; Jia, J.; Sun, Y. SpoT-mediated NapA upregulation promotes oxidative stressinduced Helicobacter pylori biofilm formation and confers multidrug resistance. Antimicrob. Agents Chemother. 2021, 65, e00152-21. [CrossRef]

10. Ye, Q.; Shao, X.; Shen, R.; Chen, D.; Shen, J. Changes in the human gut microbiota composition caused by Helicobacter pylori eradication therapy: A systematic review and meta-analysis. Helicobacter 2020, 25, e12713. [CrossRef]

11. Suthisamphat, N.; Dechayont, B.; Phuaklee, P.; Prajuabjinda, O.; Vilaichone, R.K.; Itharat, A.; Mokmued, K.; Prommee, N. AntiHelicobacter pylori, Anti-Inflammatory, Cytotoxic, and Antioxidant Activities of Mace Extracts from Myristica fragrans. Evid.-Based Complement Altern. Med. 2020, 2020, 7576818. [CrossRef] [PubMed]

12. Tran Trung, H.; Truong Thi Huynh, H.; Nguyen Thi Thuy, L.; Nguyen Van Minh, H.; Thi Nguyen, M.N.; Luong Thi, M.N. Growth-Inhibiting, Bactericidal, Antibiofilm, and Urease Inhibitory Activities of Hibiscus rosa sinensis L. Flower Constituents toward Antibiotic Sensitive- and Resistant-Strains of Helicobacter pylori. ACS Omega 2020, 5, 20080-20089. [CrossRef] [PubMed]

13. Cardoso, O.; Donato, M.M.; Luxo, C.; Almeida, N.; Liberal, J.; Figueirinha, A.; Batista, M.T. Anti-Helicobacter pylori potential of Agrimonia eupatoria L. and Fragaria vesca. J. Funct. Foods 2018, 44, 299-303. [CrossRef] 
14. Prasad, A.; Devi, A.T.; Prasad, M.N.N.; Zameer, F.; Shruthi, G.; Shivamallu, C. Phyto anti-biofilm elicitors as potential inhibitors of Helicobacter pylori. 3 Biotech 2019, 9, 53. [CrossRef]

15. Zhang, J.; Chen, Z.; Kong, J.; Liang, Y.; Chen, K.; Chang, Y.; Yuan, H.; Wang, Y.; Liang, H.; Li, J.; et al. Fullerenol Nanoparticles Eradicate Helicobacter pylori via pH-Responsive Peroxidase Activity. ACS Appl. Mater. Interfaces 2020, 12, 29013-29023. [CrossRef]

16. Yang, S.J.; Huang, C.H.; Yang, J.C.; Wang, C.H.; Shieh, M.J. Residence Time-Extended Nanoparticles by Magnetic Field Improve the Eradication Efficiency of Helicobacter pylori. ACS Appl. Mater. Interfaces 2020, 12, 54316-54327. [CrossRef]

17. Arif, M.; Sharaf, M.; Samreen; Khan, S.; Chi, Z.; Liu, C.G. Chitosan-based nanoparticles as delivery-carrier for promising antimicrobial glycolipid biosurfactant to improve the eradication rate of Helicobacter pylori biofilm. J. Biomater. Sci. Polym. Ed. 2021, 32, 813-832. [CrossRef]

18. Qureshi, N.; Li, P.; Gu, Q. Probiotic therapy in Helicobacter pylori infection: A potential strategy against a serious pathogen? Appl. Microbiol. Biotechnol. 2019, 103, 1573-1588. [CrossRef]

19. Ji, J.; Yang, H. In Vitro Effects of Lactobacillus plantarum LN66 and Antibiotics Used Alone or in Combination on Helicobacter pylori Mature Biofilm. Microorganisms 2021, 9, 424. [CrossRef]

20. Graham, D.Y.; Fischbach, L. Helicobacter pylori treatment in the era of increasing antbiotic resistance. Gut 2010, 59, 1143-1153. [CrossRef]

21. Wang, F.; Feng, J.R.; Chen, P.F.; Liu, X.P.; Ma, M.X.; Zhou, R.; Chang, Y.; Liu, J.; Li, J.; Zhao, Q. Probiotics in Helicobacter pylori eradication therapy: Systematic review and network meta-analysis. Clin. Res. Hepatol. Gastroenterol. 2017, 41, 466-475. [CrossRef] [PubMed]

22. Lv, Z.; Wang, B.; Zhou, X.; Wang, F.; Xie, Y.; Zheng, H.; Lv, N. Efficacy and safety of probiotics as adjuvant agents for Helicobacter pylori infection: A meta-analysis. Exp. Ther. Med. 2015, 9, 707-716. [CrossRef] [PubMed]

23. Jin, F.; Yang, H. Effects of Lactobacillus salivarius LN12 in Combination with Amoxicillin and Clarithromycin on Helicobacter pylori Biofilm In Vitro. Microorganisms 2021, 9, 1611. [CrossRef] [PubMed]

24. Krzyzek, P.; Grande, R.; Migdal, P.; Paluch, E.; Gosciniak, G. Biofilm Formation as a Complex Result of Virulence and Adaptive Responses of Helicobacter pylori. Pathogens 2020, 9, 1062. [CrossRef] [PubMed]

25. Franklin, M.J.; Chang, C.; Akiyama, T.; Bothner, B. New Technologies for Studying Biofilms. Microbiol. Spectr. 2015, 8, 3. [CrossRef]

26. Ge, X.R.; Cai, Y.Y.; Chen, Z.H.; Gao, S.Z.; Geng, X.W.; Li, Y.; Li, Y.; Jia, J.H.; Sun, Y.D. Bifunctional Enzyme SpoT Is Involved in Biofilm Formation of Helicobacter pylori with Multidrug Resistance by Upregulating Efflux Pump Hp1174 (gluP). Antimicrob. Agents Chemother. 2018, 62, e00957-18. [CrossRef]

27. Yu, M.; Wang, X.; Ling, F.; Wang, H.; Zhang, P.; Shao, S. Atractylodes lancea volatile oils attenuated Helicobacter pylori NCTC11637 growth and biofilm. Microb. Pathog. 2019, 135, 103641. [CrossRef]

28. Li, P.Y.; Chen, X.N.; Shen, Y.N.; Li, H.T.; Zou, Y.Q.; Yuan, G.; Hu, P.; Hu, H.Y. Mucus penetration enhanced lipid polymer nanoparticles improve the eradication rate of Helicobacter pylori biofilm. J. Control. Release 2019, 300, 52-63. [CrossRef]

29. Chen, X.N.; Li, P.Y.; Shen, Y.N.; Zou, Y.Q.; Yuan, G.; Hu, H.Y. Rhamnolipid-involved antibiotics combinations improve the eradication of Helicobacter pylori biofilm in vitro: A comparison with conventional triple therapy. Microb. Pathog. 2019, 131, 112-119. [CrossRef]

30. Smith, M.A.; Finel, M.; Korolik, V.; Mendz, G.L. Characteristics of the aerobic respiratory chains of the microaerophiles Campylobacter jejuni and Helicobacter pylori. Arch. Microbiol. 2000, 174, 1-10. [CrossRef]

31. Flemming, D.; Schlitt, A.; Spehr, V.; Bischof, T.; Friedrich, T. Iron-sulfur cluster N2 of the Escherichia coli NADH:ubiquinone oxidoreductase (complex I) is located on subunit NuoB. J. Biol. Chem. 2003, 278, 47602-47609. [CrossRef] [PubMed]

32. Tang, W.; Xiao, Y.; Long, Y.; Li, Y.; Peng, F.; Zhu, C.; He, T.; Lou, D.; Zhu, Y. Sodium fluoride causes oxidative damage to silkworm (Bombyx mori) testis by affecting the oxidative phosphorylation pathway. Ecotoxicol. Environ. Saf. 2021, 218, 112229. [CrossRef] [PubMed]

33. Bai, H.; Zhou, D.G.; Zhang, X.W.; Cao, Y.F.; Xiao, X.L.; Zhang, Y.; Yu, Y.G. The responses of Salmonella enterica serovar Typhimurium to vanillin in apple juice through global transcriptomics. Int. J. Food Microbiol. 2021, 347, 109189. [CrossRef] [PubMed]

34. Rader, B.A.; Wreden, C.; Hicks, K.G.; Sweeney, E.G.; Ottemann, K.M.; Guillemin, K. Helicobacter pylori perceives the quorumsensing molecule Al-2 as a chemorepellent via the chemoreceptor TIpB. Microbiol.-Sgm 2011, 157, 2445-2455. [CrossRef]

35. Anderson, J.; Huang, J.; Wreden, C.; Sweeney, E.; Goers, J.; Remington, S.J.; Guillemin, K. Chemorepulsion from the Quorum Signal Autoinducer-2 Promotes Helicobacter pylori Biofilm Dispersal. MBio 2015, 6, e00379-15. [CrossRef]

36. Allard-Massicotte, R.; Tessier, L.; Lecuyer, F.; Lakshmanan, V.; Lucier, J.F.; Garneau, D.; Caudwell, L.; Vlamakis, H.; Bais, H.P.; Beauregard, P.B. Bacillus subtilis Early Colonization of Arabidopsis thaliana Roots Involves Multiple Chemotaxis Receptors. $m B i o$ 2016, 7, e01664-16. [CrossRef]

37. Wistrand-Yuen, E.; Knopp, M.; Hjort, K.; Koskiniemi, S.; Berg, O.G.; Andersson, D.I. Evolution of high-level resistance during low-level antibiotic exposure. Nat. Commun. 2018, 9, 1599. [CrossRef]

38. Jutkina, J.; Marathe, N.P.; Flach, C.F.; Larsson, D.G.J. Antibiotics and common antibacterial biocides stimulate horizontal transfer of resistance at low concentrations. Sci. Total. Environ. 2018, 616-617, 172-178. [CrossRef]

39. Sato, M.; Sinha, P.K.; Torres-Bacete, J.; Matsuno-Yagi, A.; Yagi, T. Energy transducing roles of antiporter-like subunits in Escherichia coli NDH-1 with main focus on subunit NuoN (ND2). J. Biol. Chem. 2013, 288, 24705-24716. [CrossRef]

40. Lane, M.C.; O'Toole, P.W.; Moore, S.A. Molecular basis of the interaction between the flagellar export proteins FliI and FliH from Helicobacter pylori. J. Biol. Chem. 2006, 281, 508-517. [CrossRef] 
41. Wu, J.J.; Sheu, B.S.; Huang, A.H.; Lin, S.T.; Yang, H.B. Characterization of flgK gene and FlgK protein required for H. pylori colonization-from cloning to clinical relevance. World J. Gastroenterol. 2006, 12, 3989-3993. [CrossRef] [PubMed]

42. Chu, J.K.; Zhu, S.; Herrera, C.M.; Henderson, J.C.; Liu, J.; Trent, M.S.; Hoover, T.R. Loss of a Cardiolipin Synthase in Helicobacter pylori G27 Blocks Flagellum Assembly. J. Bacteriol. 2019, 201, e00372-19. [CrossRef] [PubMed]

43. Kim, N.; Marcus, E.A.; Wen, Y.; Weeks, D.L.; Scott, D.R.; Jung, H.C.; Song, I.S.; Sachs, G. Genes of Helicobacter pylori regulated by attachment to AGS cells. Infect. Immun. 2004, 72, 2358-2368. [CrossRef]

44. O'Neill, J.; Roujeinikova, A. Cloning, purification and crystallization of MotB, a stator component of the proton-driven bacterial flagellar motor. Acta Crystallogr. Sect. F Struct. Biol. Cryst. Commun. 2008, 64, 561-563. [CrossRef] [PubMed]

45. Singh, A.; Hodgson, N.; Yan, M.; Joo, J.; Gu, L.; Sang, H.; Gregory-Bryson, E.; Wood, W.G.; Ni, Y.; Smith, K.; et al. Screening Helicobacter pylori genes induced during infection of mouse stomachs. World J. Gastroenterol. 2012, 18, 4323-4334. [CrossRef]

46. Homuth, G.; Domm, S.; Kleiner, D.; Schumann, W. Transcriptional analysis of major heat shock genes of Helicobacter pylori. J. Bacteriol. 2000, 182, 4257-4263. [CrossRef]

47. Keikha, M. Is there a relationship between Helicobacter pylori vacA i1 or i2 alleles and development into peptic ulcer and gastric cancer? A meta-analysis study on an Iranian population. New Microbes New Infect. 2020, 36, 100726. [CrossRef]

48. Yang, J.; Yang, H. Transcriptome Analysis of the Clostridioides difficile Response to Different Doses of Bifidobacterium breve. Front. Microbiol. 2020, 11, 1863. [CrossRef]

49. Wang, N.; Hang, X.M.; Zhang, M.; Peng, X.Y.; Yang, H. New genetic environments of the macrolide-lincosamide-streptogramin resistance determinant $\operatorname{erm}(\mathrm{X})$ and their influence on potential horizontal transferability in Bifidobacteria. Int. J. Antimicrob. Agents 2017, 50, 572-580. [CrossRef]

50. Bindea, G.; Mlecnik, B.; Hackl, H.; Charoentong, P.; Tosolini, M.; Kirilovsky, A.; Fridman, W.H.; Pages, F.; Trajanoski, Z.; Galon, J. ClueGO: A Cytoscape plug-in to decipher functionally grouped gene ontology and pathway annotation networks. Bioinformatics 2009, 25, 1091-1093. [CrossRef] 